\title{
PRIVACY PRESERVATION MECHANISM USING CLUSTERING TECHNIQUES ON HADOOP
}

\author{
Dhanuja A.R \\ Department of CSE \\ Cambridge Institute of Technology \\ Bangalore, Karnataka, India
}

\begin{abstract}
Clustering systems have been widely received in numerous genuine information research applications, for example, customer behavior research, targeted display, advanced criminology, etc. With the information explosion in today's huge reporting period, a significant pattern for dealing with clustering into huge-scale data sets is the reappropriation of the open cloud stages. This is because distributed computing offers solid administrations with execution guarantees, but also investment funds in internal IT bases. Be that as it may, given that the data sets used for clustering may contain sensitive data, for example persistent wellness data, business information and social information, etc.,

A useful protection that safeguards the K-means cluster plan that can be productively reassigned to cloud servers. Plan enables cloud servers to legitimately group on encoded data sets, while achieving equivalent computational unpredictability and proven accuracy and grouping on decoded ones. Also, explore the safe combination of MapReduce in the plan, which makes the plan very appropriate for distributed computing conditions. A thorough safety investigation and numerical examination complete the presentation of the plan regarding safety and competition. Exploratory evaluation of a data set of 5 million items further supports the useful execution of the plan.
\end{abstract}

Keywords-MapReduce, K-Means

\section{INTRODUCTION}

Clustering is an important task of exploratory information extraction and examination of factual information, which has been universally received in numerous spaces, including human services, interpersonal organization, image research, design recognition, etc. Meanwhile, the rapid development of large amounts of information related to the analysis and extraction of current information also presents difficulties to group them in relation to volume, assortment and speed. To deftly monitor huge-scale data sets and strengthen clustering on top of them, the open cloud framework is doing important work for both execution and financial thinking. After all, Using the benefits of the open cloud definitely presents security concerns. This is because not only is a large amount of information related to information mining applications sensitive in a natural way, for example, individual welfare data, limitation information, budget information, etc., however, in addition, the Open cloud is an open domain worked by external people. [one]. For example, a promising pattern for predicting an individual's danger of illness is to pool existing patient wellness records [2], which contain sensitive patient data according to the Health Insurance Portability and Accountability Act Policy ( HIPAA) [3]. In consecuense, appropriate security assurance components must be in place while reassigning sensitive data sets to the general population cloud for pooling. The question of the protection that K-safeguards involves grouping hosts has been examined under the secure multi-meeting computation model [4] - [9], in which the owners of transmitted data sets collaborate to group without revealing their own sets of data with each other. In the multi-party environment, each meeting has a variety of information and wants to work together with others in a way to safeguard protection to improve grouping accuracy. In an unexpected way, the data set in the pooled redistribution is normally claimed by a solitary substance, which is intended to limit neighborhood computation by designating the cluster assignment to an external cloud server. Furthermore, existing multi-party structures constantly rely on incredible but expensive cryptographic natives (e.g., Secure Circuit Evaluation, Homomorphic Encryption, and Negligent Sharing) to achieve secure cooperative computation across multiple meetings, and are wasteful for data sets to big scale. The way, these multi-party structures are not viable for protection by safeguarding the reappropriation of the grouping. A different line of research that aims at productive security protection bundling is to use separation to protect information nuisance or information change to encode data sets [10], [11]. By the way, the use of information nuisance and information exchange to bundle security protection may not achieve sufficient protection and guarantee accuracy [12], [13]. For example, enemies who obtain a pair of decoded information 


\section{International Journal of Engineering Applied Sciences and Technology, 2020 \\ Vol. 5, Issue 4, ISSN No. 2455-2143, Pages 323-330 \\ Published Online August 2020 in IJEAST (http://www.ijeast.com)}

records in the dataset will have the option to retrieve secured rest records by information change [12]. Lately, the redistribution of the $\mathrm{K}$-imply cluster is contemplated in reference [14] through the use of homomophic encryption and the request save list. Be that as it may, the homomophic cipher used in [14] is not verified as mentioned in ref [15]. Also, due to the cost of relatively expensive homomophic encryption, reference [14] is productive only for small data sets, eg less than 50. 000 information objects. Another conceivable possibility to achieve the clustering of K-protection saving implications is to extend the existing security by saving the $\mathrm{K}$ nearest neighbor (KNN) search plans [16] - [18]. Unfortunately, these protections that safeguard KNN search plans are restricted by defenselessness to direct examination assaults [16], support for up to two data measurements [17], or lack of accuracy [18]. Also, KNN is a lone round search task, however $\mathrm{K}$-imply clustering is an iterative procedure that requires updating of clustering bindings that depend on the entire data set after each clustering round. Considering productive help on huge-scale data sets, these update forms additionally they must be reassigned to the cloud server to save protection. Aside from security assurance, there are two other main considerations in reappropriating the K-pool involves: pooling efficiency and pooling accuracy. In particular, a down-to-earth $\mathrm{K}$ redistribution to preserve privacy implies that clustering will be effectively parallelized, which is significant under distributed computing conditions to ensure execution on huge-scale data sets. Meanwhile, the computational expense of the owner of the dataset will be limited, that is, the owner is responsible for the arrangement of the framework as well as light collaborations with servers in the cloud. While several MapReduce-based K-implies plans have been dealing with large-scale data sets in parallel [19] [21], none of them think about ensuring security for the redistributed data set. Additionally, the security assurance offered in a K-imply redistributed configuration will have a slight

(Also, not even) impact on grouping accuracy. This is because accuracy is the key factor in deciding the nature of a grouping calculation. As far as one might know, there is no current $\mathrm{K}$ implicit K-implicit security savings reappropriation plan that can achieve virtually identical productivity and, furthermore, precision in grouping unprotected data sets.

K-saving functional protection involves a clustering plan for large-scale data sets, which can be competently redistributed to open cloud servers. At the same time, the plot meets the prerequisites for protection, competence, and accuracy discussed above. Specifically, a novel cipher that relies on the difficult problem to learn with error (LWE) [22], which achieves the estimation of privacy preservation similarities of information questions directly on ciphertext. In light of the encryption plot, further develop the entire K-bundling process in a way that preserves privacy, in which cloud servers simply zoom in on encrypted data sets and will perform all tasks. undeciphered. Also, exceptionally consolidate MapReduce [23] in the plan with security insurance, and along these lines, fully improve the clustering performance under distributed computing conditions. Give the plan a thorough review with regard to safety and competition.

I also implemented a prototype schema in the Microsoft Azure cloud. The results of a comprehensive assessment of more than 5 million objects show that privacy-preserving clustering is efficient, scalable, and accurate. Specifically, compared to grouping K-means over unencrypted data sets, the scheme achieves the same precision, as well as comparable computational performance and scalability.

\section{RELATED WORK}

[1] Darcy A. et al. Prediction of the possibility of singular infection "that depends on the history of the restoration. The enormous expense of the social security, in particular for the incessant treatment of diseases, is fast becoming unmanageable". This emergency has sparked the momentum towards protective medication, where the main concern is to perceive the danger of disease and act as soon as possible. Be that as it may, widespread testing is not productive in time or cost. CARE, a collaborative evaluation and recommendation engine, that relies only on a patient's restorative history using ICD-9-CM codes to anticipate future disease hazards. CARE uses community-oriented segregation to anticipate each patient's major disease hazards based on their own medical history and that of comparable patients. In addition, they represent an iterative variant, ICARE, that merges company ideas for better execution. These story frames do not require specific data and give expectations to ailments of many kinds in a lonely career. Present exploratory results in a Medicare data set, demonstrating that CARE and ICARE work well to detect future infection hazards. These story frames do not require specific data and give expectations to ailments of many kinds in a lonely career. Present exploratory results on a Medicare dataset, showing that CARE and ICARE work well to detect future infection dangers. These story frames do not require specific data and give expectations to ailments of many kinds in a lonely career. Present exploratory results in a Medicare data set, demonstrating that CARE and ICARE work well to detect future infection hazards.

[2] Jaideep Vaidya et al. K-privacy saving involves grouping over vertically distributed information. Protection and security concerns can anticipate information sharing, bankrupting information mining companies. Disclosure of disseminated information, provided it is done effectively, can reduce this problem. The key is to obtain legitimate results, while granting certifications on the (non) disclosure of information. Presenting a technique for k-involves grouping when multiple destinations contain multiple properties for a typical arrangement of elements. Each site learns the set of each 


\section{International Journal of Engineering Applied Sciences and Technology, 2020 Vol. 5, Issue 4, ISSN No. 2455-2143, Pages 323-330 \\ Published Online August 2020 in IJEAST (http://www.ijeast.com)}

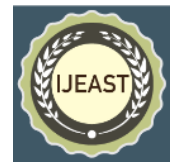

element, except it does not adapt anything about the properties in different locales

[3] Geetha Jagannathan et al. The privacy savings in circulation $\mathrm{k}$ involves grouping subjectively divided information. Advances in PC system management and database innovations have enabled the assortment and capacity of vast amounts of information. Information mining can remove important information from this information, and associations have understood that they can regularly get better results by pooling their information. However, the information collected may contain sensitive or private data about associations or their clients, and security concerns are compounded if the information is shared between different organizations. Distributed information mining is concerned with calculating models from the information that circulates among numerous members. The search for transmitted information of safety savings seeks to take into account the useful calculation of such models without the coordination meetings discovering any of their individual information things. The two compromises in security saving information mining. To begin, introduce the idea of subjectively distributed information, which is a speculation of both a level plane and vertically divided information. Second, provide a competent protection savings convention for clustering $\mathrm{k}$ implies in the context of self-asserting information divided. which is a speculation of information both on a level plane and in vertical plots. Secondly, provide a competent protection saving convention for $\mathrm{k}$-implies grouping in the context of self-asserting divided information. which is a speculation of information both on a level plane and in vertical plots. Second, provide a competent protection saving convention for $\mathrm{k}$ implies grouping in the context of self-asserting information divided.

[4] Paul Bunn et al. Two-part safe k-clustering implies The kmeans clustering problem is one of the most investigated topics in information mining to date. With the focus on conventions that have proven fruitful in realizing unique database pools, the focus has lately moved to the topic of how to extend single database conventions to a different database configuration. Several efforts have been made to date to make explicit multiple k-implication grouping conventions that ensure the security of all databases except as indicated by the standard cryptographic meanings of "safeguard", so far all of these efforts have failed to provide sufficient security.

They represent a two-part k-means clustering protocol that ensures security and is more proficient than using a general multi-part "compiler" to perform a similar mapping. Specifically, a fundamental compromise of result is an approach to effectively record multiple clustering cycles of kimplies without discovering the intermediate qualities. To accomplish this, demonstrate two systems: perform a bipartite division and consistently test on an arbitrary space size from a dark; the subsequent Split Protocol and Random Value Protocol are useful for any convention that requires the safe calculation of a remnant or irregular exam. The strategies can be recognized depending on the presence of any semantically secure homomorphic encryption conspiracy. For robustness, portray the conspiracy-dependent convention of Paillier's homomorphic cipher. Also, demonstrate that the convention is productive when it comes to correspondence, staying focused on existing conventions that neglect safe protection.

[5] Mahir Can Doganay, et al. Distributed security safeguarding k-involves bundling with the mystery exchange of additional substances. Ongoing concerns about protection issues prompted information mining scientists to create techniques to mine information while safeguarding the safety of people. In any case, current strategies to protect information mining security experience the deleterious effects of high matching and computational overhead that are restrictive considering even a modest database size. In addition, the strategies harbor exact suspicions about the included meetings that must be flexible to reflect the needs of the current reality. The focus on an appropriate situation where information is vertically distributed among numerous destinations and included locations may want to group without discovering their nearby databases. For this configuration, another convention for $\mathrm{k}$-safeguarding protection involves clustering that depends on the mystery exchange of added substances. Show that the new convention is safer than the best in class. Trials directed at genuine and designed collections of information show that, in reasonable situations, the cost of matching and computing the convention is not exactly the cutting edge that is urgent for information mining applications. Show that the new convention is safer than the best of its kind. Trials targeting genuine and engineered information collections show that, in reasonable situations, the cost of convention matching and computation is not exactly the cutting edge that is urgent for information mining applications. Show that the new convention is safer than the best of its kind. Trials targeting genuine and engineering informational collections show that, in reasonable situations, the cost of convention and convention calculation is not exactly the cutting edge that is urgent for information mining applications. Show that the new convention is safer than the best of its kind. Trials targeting genuine and engineering informational collections show that, in reasonable situations, the cost of convention and convention calculation is not exactly the cutting edge that is urgent for information mining applications. Show that the new convention is safer than the best of its kind. Trials targeting genuine and engineering informational collections show that, in reasonable situations, the cost of convention and convention calculation is not exactly the cutting edge that is urgent for information mining applications.

[6] Jun Sakuma et al Large-scale k-means clustering with user-centric privacy preservation A k-means grouping is 


\section{International Journal of Engineering Applied Sciences and Technology, 2020 \\ Vol. 5, Issue 4, ISSN No. 2455-2143, Pages 323-330 \\ Published Online August 2020 in IJEAST (http://www.ijeast.com)}

introduced with a new concept of privacy preservation, usercentered privacy preservation. Marco, users can perform data mining using their private information by storing it on their local storage. After calculation, they get only the mining result without revealing private information to others. In most cases, the number of parties that can join conventional privacy preservation data mining is assumed to be only two. In the framework, suppose that a large number of parties join the protocol; therefore, not only scalability is important, but also asynchronism and fault tolerance. With this in mind, a k-mean algorithm combined with a decentralized cryptographic protocol and a gossip-based protocol.

[7] Xun Yi v Equally Contributing Privacy Conservation kmeans grouping on vertically divided data.

In recent years, there have been numerous attempts to extend the k-means clustering protocol for a single database to a distributed multiple database configuration while keeping each data site private. Current solutions for (either two or more) clusters of multipart k-means, based on one or more secure two-part computation algorithms, are not equally contributory, in other words, each part does not contribute equally to the clustering of $\mathrm{k}$-socks. This can lead to a perfidious attack in which one party who finds out the result before other parties tells a lie about the result to other parties. We present an equally contributory multi-part k-means clustering protocol for vertically divided data, in which each part contributes equally to the grouping of k-means. The protocol is based on the ElGamal, Jakobsson and Juels encryption scheme

[8] Stanley RM Oliveira et al preserving privacy grouping by data transformation.

Preserving people's privacy when data is shared for grouping is a complex issue. The challenge is how to protect the underlying data values subject to grouping without compromising the similarity between the objects under analysis. Revisit a family of Geometric Data Transformation (GDTM) methods that distort numeric attributes through translations, scales, rotations, or even by combining these geometric transformations. This approach was designed to address privacy-preserving clustering in scenarios where data owners must not only comply with privacy requirements, but also ensure valid clustering results. Please offer a detailed picture,

[9] Dongxi Liu et al The privacy of the k-redistributed clustering implies.

It calls for an association to redistribute its information review to a specialized cooperative that has innovative stages and advanced research skills. However, the association (owner of the information) may have concerns about the protection of your information. Presenting a strategy that allows the owner of the information to encode their information with a homomorphic encryption conspiracy and for the specialized organization to perform k-involves bundling directly on the encoded information. Be that as it may, since the ciphertexts that are produced due to homomorphic encryption do not save the request for separations between information items and group approaches,

[10] Yongge Wang et al Notes on two fully homomorphic cipher plans without bootstrapping.

Lately, the IACR ePrint file published two fully homomorphic encryption plans without bootstrapping. Bear in mind, show that these plans are inconsistently uncertain. Also, further show that the cipher graphics in CCS 2012 and the cipher conspire in ASIACCS 2014 are also not unstable.

[11] Wai Kit Wong, et al. Secure calculation of knn in encrypted databases.

Specialized organizations like Google and Amazon are moving into the SaaS (Software as a Service) business. They transform their gigantic framework into a distributed computing condition and forcibly recruit organizations to run applications at their base. To authorize security and safety in equally to the clustering of $\mathrm{k}$-socks. This can lead to a perfidious attack in which one party who finds out the result before other parties tells a lie about the result to other parties. We present an equally contributory multi-part k-means clustering protocol for vertically divided data, in which each part contributes equally to the grouping of $\mathrm{k}$-means. The protocol is based on the ElGamal, Jakobsson and Juels encryption scheme

[8] Stanley RM Oliveira et al preserving privacy grouping by data transformation.

Preserving people's privacy when data is shared for grouping is a complex issue. The challenge is how to protect the underlying data values subject to grouping without compromising the similarity between the objects under analysis. Revisit a family of Geometric Data Transformation (GDTM) methods that distort numeric attributes through translations, scales, rotations, or even by combining these geometric transformations. This approach was designed to address privacy-preserving clustering in scenarios where data owners must not only comply with privacy requirements, but also ensure valid clustering results. Please offer a detailed picture,

[9] Dongxi Liu et al The privacy of the k-redistributed clustering implies.

It calls for an association to redistribute its information review to a specialized cooperative that has innovative stages and advanced research skills. However, the association (owner of the information) may have concerns about the protection of 


\section{International Journal of Engineering Applied Sciences and Technology, 2020 \\ Vol. 5, Issue 4, ISSN No. 2455-2143, Pages 323-330 \\ Published Online August 2020 in IJEAST (http://www.ijeast.com)}

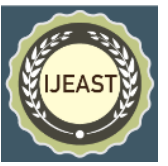

your information. Presenting a strategy that allows the owner of the information to encode their information with a homomorphic encryption conspiracy and for the specialized organization to perform k-involves bundling directly on the encoded information. Be that as it may, since the ciphertexts that are produced due to homomorphic encryption do not save the request for separations between information items and group approaches,

[10] Yongge Wang et al Notes on two fully homomorphic cipher plans without bootstrapping.

Lately, the IACR ePrint file published two fully homomorphic encryption plans without bootstrapping. Bear in mind, show that these plans are inconsistently uncertain. Also, further show that the cipher graphics in CCS 2012 and the cipher conspire in ASIACCS 2014 are also not unstable.

[11] Wai Kit Wong, et al. Secure calculation of knn in encrypted databases.

Specialized organizations like Google and Amazon are moving into the SaaS (Software as a Service) business. They transform their gigantic framework into a distributed computing condition and forcibly recruit organizations to run applications at their base. To authorize security and safety ultimate goal being guaranteed that $E(G)$ contains the answer for the SNN query. The techniques offer an adaptive trade-off between productivity and cost of matching, and are as secure as the encryption diagram E used to encrypt the question and the database, where E can be any established encryption plan. plan new SNN strategies by asking the server, given only $\mathrm{E}$ (q) and $E(D)$, to restore a relevant (encoded) packet $E(G)$ from $E$ (D) (i.e. $G \subseteq D$ ), with the goal final $E(G)$ is guaranteed to contain the response for the SNN query. The techniques offer an adaptive trade-off between productivity and cost of correspondence,

[13] Sen Su, et al. Protecting the privacy of top-k spatial slogan questions on redistributed databases.

Study the issue of security protecting the problem of investigation of the space setpoint top-kk under redistributed conditions. Existing research is mainly focused on the protection plan that safeguards the plans for spatial or slogan queries, and cannot be applied to unravel the problem of the issue of the spatial safety saving slogan. To address this issue, present a novel protection that preserves the top-kk conspire spatial reference phrase question. Specifically, build a coded tree file to further protect, saving top-kk spatial citation questions, where spatial and printed information is coded in a tight manner. To look with the hardcoded tree list, two convincing systems for proximity calculations between queries and tree centers under encryption. Careful research shows the legitimacy and security of the plan.
[14] Weizhong Zhao et al. Parallel k-implies mapreducedependent clustering. Information grouping has received high consideration in numerous applications, for example information mining, report retrieval, image splitting, and characterization of samples. The ever-increasing volumes of data that are developed with the advancement of innovation make gathering together a large amount of information a difficult task. To manage the problem, many analysts try to structure effective parallel grouping calculations. A clustering calculation of $\mathrm{k}$-implies in parallel that it depends on MapReduce, which is a simple but amazing parallel programming procedure.

[15] Xiaoli Cui, et al. Optimized the enormous amount of information k-involves clustering using mapreduce.

The grouping test is one of the most used pieces of information when preparing calculations. For 50 years, K -imp has remained the best known grouping calculation as a result of its simplicity. Lately, as the volume of information continues to grow, some analysts are turning to MapReduce to get the elite. Be that as it may, MapReduce is unacceptable for repeated calculations attributable to repeated occupancy reset times, heavy reading of information, and reordering. Addressing the problems of preparing large-scale information using $\mathrm{K}$-involves clustering computation and proposing a novel management model in MapReduce to eliminate cycle dependency and acquire superior. The decomposed and updated thinking. Extensive analyzes on the group show that the techniques are competent,

[16] Zvika Brakerski, et al. Ciphers encrypted in homomorphic encryption based on lwe.

The Peikert-Vaikuntanathan-Waters (PVW) technique for pressing numerous plaintext components into a single Regevtype ciphertext can be used to perform homomorphic SIMD tasks in filled ciphertext. This offers an option in contrast to the Smart-Vercauteren (SV) ciphertext packaging method that relies on the CRT-polynomial. While the SV strategy is only material for planes that depend on the ring-LWE (or different suspected hardness in perfect cross sections), the PVW technique can be used in the same way for cryptosystems whose safety depends on the standard LWE (or a lot more broadly than the hardness of "General-LWE"). Although using the PVW strategy with LWE-based plans results in more horrible asymptotic productivity than using the SV procedure with ring-LWE plans, in any case, the simplicity of this technique may offer some reasonable favorable circumstances. . Also, the two procedures can be used in conjunction with "general-LWE" plans, recommending one more offset that can be improved for various environments. 


\section{International Journal of Engineering Applied Sciences and Technology, 2020 Vol. 5, Issue 4, ISSN No. 2455-2143, Pages 323-330 \\ Published Online August 2020 in IJEAST (http://www.ijeast.com)}

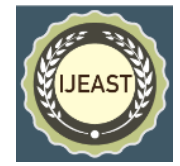

[17] Jeffrey Dean et al Mapreduce: simplified data processing in large groups.

MapReduce is a programming model and associated implementation for processing and generating large data sets. Users specify a map function that processes a key / value pair to generate a set of intermediate key / value pairs, and a reduce function that merges all intermediate values associated with the same intermediate key. Many real-world tasks are expressible in the model. Programs written in functional style are automatically parallelized and run on a large group of basic machines. The runtime system takes care of the details of partitioning the input data, scheduling the execution of the program on a set of machines, handling machine faults, and managing the required inter-machine communication. This allows programmers without experience with distributed and parallel systems to easily use the resources of a large distributed system. The MapReduce implementation runs on a large group of basic machines and is highly scalable: a typical MapReduce calculation processes many terabytes of data on thousands of machines. Developers find the system easy to use: Hundreds of MapReduce programs have been deployed and over a thousand MapReduce jobs run on Google clusters every day.

[18] Sabrina De Capitani di Vimercati, et al Excessive encryption: managing evolution of access control in outsourced data.

Data outsourcing is emerging today as a successful paradigm that enables users and organizations to exploit external services for resource allocation. A crucial issue that needs to be addressed in context concerns the enforcement of selective authorization policies and the support of policy updates in dynamic scenarios.

Present a novel solution for the application of access control and the management of its evolution. It is based on the application of selective encryption as a means to enforce authorizations. Two layers of encryption are imposed on data: the inner layer is imposed by the owner to provide initial protection, the outer layer is imposed by the server to reflect policy changes. The combination of the two layers provides an efficient and robust solution. A model, an algorithm for managing the two layers and an analysis to identify and thus counteract the possible risks of information exposure.

[19] Jiawei Yuan et al. Protecting the privacy of extended neural system learning became pragmatic with distributed computing.

To improve the accuracy of the learning outcome, in practice, numerous meetings can work together directing the joint learning of the Back-Propagation neural system on the association of its separate information indices. During this procedure, no collection needs to reveal your private information to other people. Existing plans that support this type of community-oriented learning are restricted in the method for the information pack or simply think of two meetings. There is no response that allows at least two meetings, each with a self-affirmatively parceled information index, to cooperatively lead learning. Problem using distributed computing intensity. Each meeting encrypts your private information locally and transfers the encrypted texts to the cloud. The cloud at that point executes most of the tasks related to learning calculations on encrypted texts without knowing the first private information. By safely downloading expensive activities to the cloud, keep the calculation and correspondence costs for each meeting negligible and free for the number of members. To aid adaptive activities on ciphertext, receive and adapt the "doubly homomorphic" BGN cipher calculation for the multipart environment. Numerical examination and item cloud analytics show the plan to be safe, effective, and accurate. Keep the calculation and correspondence costs at each meeting negligible and free for the number of members. To aid adaptive activities on ciphertext, receive and adapt the "doubly homomorphic" BGN cipher computation for the multipart environment. Numerical examination and item cloud analytics show the plan to be safe, effective, and accurate. Keep the calculation and correspondence costs at each meeting insignificant and free for the number of members. To aid adaptive activities on ciphertext, receive and adapt the "doubly homomorphic" BGN cipher calculation for the multipart environment. Numerical review and item cloud analytics show the plan to be safe, effective, and accurate. Numerical examination and item cloud analytics show the plan to be safe, effective, and accurate. Keep the calculation and correspondence costs at each meeting insignificant and free for the number of members. To aid adaptive activities on ciphertext, receive and adapt the "doubly homomorphic" BGN cipher calculation for the multipart environment. Numerical review and item cloud analytics show the plan to be safe, effective and accurate. Numerical review and item cloud analytics show the plan to be safe, effective, and accurate. Keep the calculation and correspondence costs at each meeting insignificant and free for the number of members. To aid adaptive activities on ciphertext, receive and adapt the "doubly homomorphic" BGN cipher calculation for the multipart environment. Numerical review and item cloud analytics show the plan to be safe, effective, and accurate.

[20] Ning Cao, et al. Privacy protection question about information organized in graphics encoded in distributed computing.

In the developing worldview of distributed computing, information owners are progressively inspired to redistribute their puzzling information in board frames from places close to the enterprise open cloud to achieve extraordinary adaptability and monetary investment funds. For the idea of customer security, sensitive information must be encrypted before being redistributed, which makes the successful use of 


\section{International Journal of Engineering Applied Sciences and Technology, 2020 \\ Vol. 5, Issue 4, ISSN No. 2455-2143, Pages 323-330 \\ Published Online August 2020 in IJEAST (http://www.ijeast.com)}

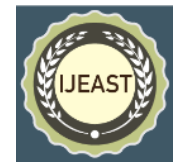

the information a test task. Just for the sake of it, characterize and address the problem of the protection issue of protection over information organized in encoded graphics in distributed computing (PPGQ), and develop many demanding security needs so that an information use framework in the cloud so sure to become a reality. The work uses the "sift and check" standard. Pre-create an item-based list to provide highlightrelated data on each coded information box, and then choose the effective internal item as the pruning apparatus to complete the screening system. To address the difficulty of supporting diagram query without protection breaks, safe internal element calculation procedure, and then improve it to achieve different security prerequisites under the known-base risk model. and then choose the effective internal element as the pruning apparatus to complete the screening system. To address the difficulty of supporting diagram query without protection breaks, safe internal element calculation procedure, and then improve it to achieve different security prerequisites under the known-base risk model. and then choose the effective inner element as the pruning apparatus to complete the screening system. To address the difficulty of supporting diagram query without protection breaks, safe internal element calculation procedure, and then improve it to achieve different security prerequisites under the known-base risk model.

[21] Jiawei Yuan et al, Efficient biometric signature test that preserves privacy in distributed computing.

The biometric distinction test is a reliable and useful method to recognize people. Powerful receipt of distinctive biometric evidence requires strong security insurance against potential abuse, mishap, or theft of biometric information. Existing methods for security guarding recognizable biometric evidence rely primarily on regular crypto natives, for example homomorphic encryption and sloppy sharing, which inevitably familiarize with the huge overhead of the framework and are not relevant to large-scale applications. A recognizable biometric test plan that saves novel protection that achieves productivity by misusing the intensity of distributed computing. Plan, the biometric database is encrypted and redistributed to servers in the cloud. To perform a recognizable biometric test, the database owner produces an accreditation for the competitor's biometric feature and sends it to the cloud. Cloud servers perform discoverable tests on the encoded database using accreditation and return the result to the owner. During identification, the cloud does not adapt anything about the first private biometric information. Since distinctive test tasks are securely redistributed to the cloud, real-time matching / computing costs on the owner's side are negligible. An intensive examination shows that the plan is safe and offers a more significant level of protection insurance than related agreements, for example, searching $\mathrm{kNN}$ in encrypted databases. Genuine Amazon cloud research, on databases of various sizes,
[22] Rafail Ostrovsky et al., The feasibility of lloyd-type strategies for the k-implicate issue.

Explore variations of the Lloyd's heuristic to group highdimensional information trying to clarify its prevalence (50 years after its presentation) among specialists, and thus propose updates in its application. Legitimize a grouping rule for informational indexes. Variations of Lloyd's heuristic that quickly lead to a provable approximation to ideal grouping arrangements when applied to well-grouped examples. This is the main guarantee of execution for a variation of the Lloyd heuristic. The provision of a guarantee on the quality of performance does not detract from speed: part of the calculations compete to be faster than the currently used variations of Lloyd's technique. Also, the different calculations are faster in well-grouped occurrences than in late estimate calculations, maintaining comparative certifications on the quality of the grouping. The primary algorithmic commitment is a novel probabilistic seeding process for the initial setup of a Lloyd-type emphasis.

\section{CONCLUSION}

Preserving privacy for data analysis is a challenging research topic due to ever-increasing volumes of data sets, requiring intensive research. Each privacy preservation technique has its own importance. Data encryption and anonymity are widely adopted ways to combat privacy breach. However, encryption is not suitable for the data that is being processed and shared. Anonymizing big data and managing anonymized data sets remain challenges for traditional anonymization approaches. Privacy-preserving data mining arises for two vital needs: data analytics to provide better services and to ensure the privacy rights of data owners. Significant efforts have been made to address these needs. An overview of recent approaches to privacy preservation was presented. Privacy guarantees,

\section{FUTURE WORK}

The other machine learning techniques for better comparison of results. Add more security and privacy with advanced encryption algorithms.

\section{REFERENCES}

[1] European Agency for Network and Information Security. Cloud Computing Security Risk Assessment. https://www.enisa.europa.eu/activities/riskmanagement/files/d eliverables/cloud-computing-risk-assessment.

[2] Xun Yi and Yanchun Zhang. K-grouping means equally contributor preserving privacy over vertically divided data. Inf. Syst., 38 (1): 97-107, March 2013. 
[3] Dongxi Liu, Elisa Bertino and Xun Yi. Privacy of the subcontracted k-medias pool. In Proceedings of the 9th ACM Symposium on Information Security, Computing and Communications, ASIA CCS '14, pages 123-134, New York, NY, USA, 2014. ACM.

[4] Yongge Wang. Notes on two fully homomorphic cipher schemes without bootstrapping. Cryptology ePrint Archive, Report 2015/519, 2015.

[5] B. Yao, F. Li and X. Xiao. Safe nearest neighbor revisited. In Data Engineering (ICDE), 29th International Conference of IEEE 2013, pages 733-744, April 2013.

[6] Sen Su, Yiping Teng, Xiang Cheng, Yulong Wang, and Guoliang Li. Privacy-preserving top-k spatial keyword queries over outsourced databases. In Proceedings of the 20th International Conference on Database Systems for Advanced Applications, DASFAA'15, pages 589-608, 2015.

[7] Bahman Bahmani, Benjamin Moseley, Andrea Vattani, Ravi Kumar, and Sergei Vassilvitskii. Scalable K means ++. 5 (7): 622-633, March 2012.

[8] Xiaoli Cui, Pingfei Zhu, Xin Yang, Keqiu Li, and Changqing Ji. Optimized clustering of k-means big data using mapreduce. J. Supercomput., 70 (3): 1249-1259, December 2014.

[9] Zvika Brakerski, Craig Gentry, and Shai Halevi. Encrypted texts packed in lwe-based homomorphic encryption. At the XVI International Conference on Practice and Theory in Public Key Cryptography (PKC), pages 1-13, February 2013.

[10] Jiawei Yuan and Shucheng Yu. Privacy preserving backward propagation neural network learning made practical with cloud computing. IEEE Transactions in Parallel and Distributed Systems, 25 (1): 212-221, 2014.

[11] Ning Cao, Zhenyu Yang, Cong Wang, Kui Ren, and Wenjing Lou. Privacy-preserving query on encrypted data with graphics structure in cloud computing. In Distributed Computing Systems (ICDCS), 31st International Conference 2011, pages 393-402, 2011.

[12] Jiawei Yuan and Shucheng Yu. Efficient biometric identification that preserves privacy in cloud computing. In 2013 Proceedings IEEE INFOCOM (INFOCOM'2013), pages 2652-2660, Turin, Italy, April 2013.

[13] Ackerman Margareta, Ben-David Shai, Branzei Simina, and Loker David. Weighted grouping. pages 858-863, 2012.
[14] Rafail Ostrovsky, Yuval Rabani, Leonard J. Schulman, and Chaitanya Swamy. The effectiveness of lloyd-type methods for the k-means problem. J. ACM, 59 (6): 28: 1-28: 22, January 2013. 